\title{
Intense $\gamma$-ray generation for a polarized positron beam in a linear collider
}

\author{
Y. Miyahara \\ SPring-8, Mikazuki, Sayo-gun, Hyogo 679-5198, Japan \\ (Received 3 June 2001; published 28 December 2001)
}

\begin{abstract}
$\gamma$-ray generation by Compton backscattering in an optical lens series with periodic focal points is considered to produce a polarized positron beam for a linear collider. The lens series is composed of 20 unit cells with a length of $210 \mathrm{~mm}$. Each lens has a hole to pass an electron beam with an energy of $5.8 \mathrm{GeV}$ and the generated $\gamma$ rays. It is shown by diffraction analysis that laser beam loss in the series is very small, and the beam size is periodically reduced to $26 \mu \mathrm{m}$. Electron beam size is reduced to $34 \mu \mathrm{m}$ in a superconducting solenoid with a field of $15 \mathrm{~T}$. To get a required $\gamma$-ray yield of $7 \times 10^{15} \gamma / \mathrm{s}$, only one circularly polarized $\mathrm{CO}_{2}$ laser source with a power of $24 \mathrm{~kW}$ is needed.
\end{abstract}

DOI: 10.1103/PhysRevSTAB.4.121001

PACS numbers: 07.85.-m

\section{INTRODUCTION}

A longitudinally polarized positron beam for a linear collider can be produced by colliding circularly polarized $\gamma$ rays with a metal target, and such $\gamma$ rays can be generated, for instance, by passing a high energy electron beam through a helical undulator [1]. The polarized $\gamma$ rays can also be generated by using a circularly polarized electromagnetic wave such as an intense laser and a microwave in place of the undulator or by Compton backscattering [1,2]. As a practical consideration of generating polarized $\gamma$ rays for the Japan Linear Collider, it was recently proposed to use 40 sets of $3.2 \mathrm{~kW} \mathrm{CO}$ lasers to provide the power into 40 sets of parabolic mirrors for the collision [3], which is referred to here as Proposal I.

Since good optical lenses are commercially available at a wavelength of $\lambda_{s}=10.6 \mu \mathrm{m}$, in this work we pass a pulsed laser beam through an optical lens series with periodic focal points, where the collision is made with a bunched electron beam. In a simple ideal case of no laser beam loss through the lens series and the same sizes of electron and laser beams with those of Proposal I, only one laser source of $4.7 \mathrm{~kW}$ with 40 unit cells is needed for the required $\gamma$-ray yield $Y=7 \times 10^{15} \gamma / \mathrm{s}$. In this paper,

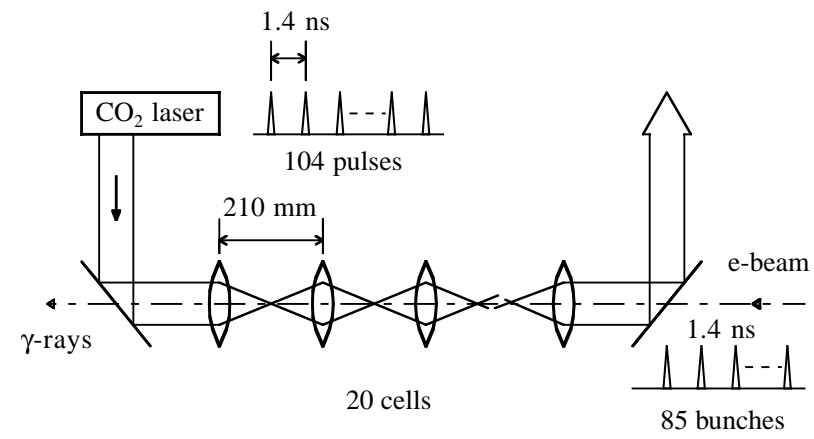

FIG. 1. Schematic structure of the present optical system. A pulsed laser beam passes through a lens series with periodic focal points and collides with a bunched electron beam to generate $\gamma$ rays. we discuss the transmission and profile of the laser beam in a lens series as well as the focusing of the electron beam, and estimate the laser power required for the $\gamma$-ray yield.

Figure 1 shows the schematic structure of the present optical system, which is composed of $N_{c}=20$ unit cells of focusing lenses with a cell length $L_{c}=210 \mathrm{~mm}$. Electron bunches and laser pulses in trains are both separated by $1.4 \mathrm{~ns}$ or $420 \mathrm{~mm}$. The electron beam energy is $5.8 \mathrm{GeV}$ to generate $\gamma$ rays with $60 \mathrm{MeV}$ in maximum by the collision.

\section{LASER BEAM OPTICS}

\section{A. Aberration}

In geometric optics, a light emitted from a point through a lens is focused at a point if the light ray is close to the beam axis. The light ray in off axis, however, goes to a different position or spreads out in the focal plane, thus an aberration is induced. Among several kinds of aberrations, only the spherical aberration is considered here, since the profile in the focal plane is very small in the present case.

The spherical aberration depends on the curvature of the lens surface. Figure 2 shows the aberrations $W_{1 s}$ and $W_{2 M}$ calculated for a conventional lens and a doublet with a form of Meniscus lens, respectively. As shown in the figure, the aberration can be fitted by $W(\mu \mathrm{m})=\xi v^{4}$, with $v(\mathrm{~mm})$ the distance from the beam axis. We get $\xi=-9.06 \times 10^{-4}$ and $-2.12 \times 10^{-4}$ for $W_{1 s}$ and $W_{2 M}$, respectively. As discussed later, the effects of aberration on the profile even with $\xi= \pm 9.06 \times 10^{-4}$ are rather large. In addition, the effects of aberration on the profile are algebraically accumulated in the lens series, so that it is necessary to reduce the aberration significantly.

Aberrations $W_{1 s}$ and $W_{2 M}$ are negative and become larger for a larger $v$. If the radius of the surface curvature is gradually increased for a larger $v$, the aberration can be reduced in principle to zero, as shown by $W_{1 m}$ in the figure. If the aberration is reduced to $\xi \approx \pm 1.0 \times 10^{-4}$, for instance, the total aberration in the 20 cells might be reduced to about $\xi \approx \pm 5 \times 10^{-4}$. 


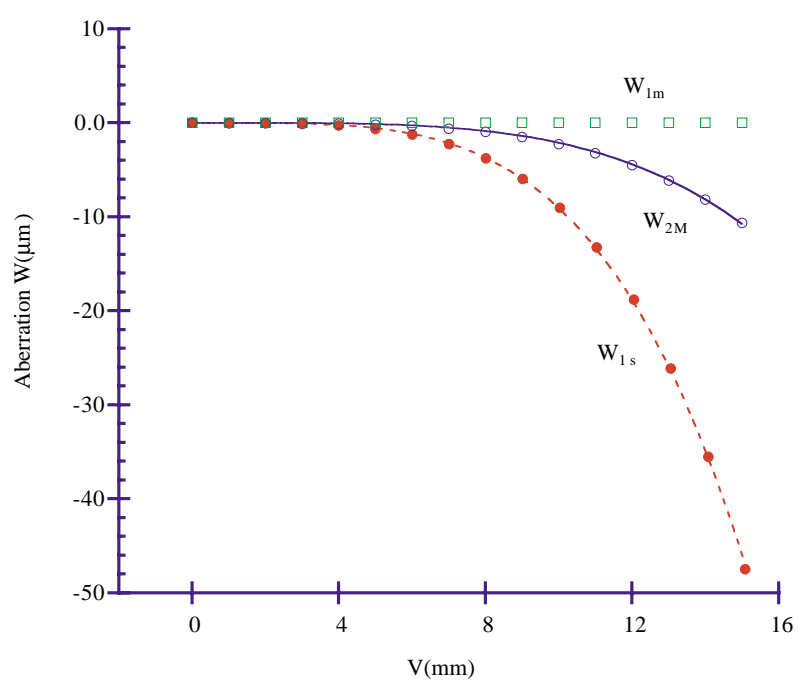

FIG. 2. (Color) Aberrations $W_{1 s}, W_{2 M}$, and $W_{1 m}$ for a conventional lens, a doublet with a form of Meniscus lens, and a modified surface lens, respectively.

\section{B. Diffraction analysis}

As shown in Fig. 3, the initial laser beam with a Gaussian distribution with size $\sigma_{r 00}=2 \mathrm{~mm}$ is converted with a circular cone to a hollow beam with a hollow radius $r_{0}=3 \mathrm{~mm}$, and injected into the lens series. Each lens has an aperture $D_{1}\left(=2 r_{2}\right)=30 \mathrm{~mm}$ with a hole diameter $D_{h}\left(=2 r_{1}\right)=6 \mathrm{~mm}$ to pass the electron beam and the generated $\gamma$ rays with an energy higher than $1 \mathrm{MeV}$. The amplitude function of the hollow beam is given by

$$
\begin{array}{r}
U_{0}(r)=\frac{1}{\sqrt{2 \pi} \sigma_{r 00}} \sqrt{\frac{r-r_{0}}{r}} \exp \left[-\frac{\left(r-r_{0}\right)^{2}}{4 \sigma_{r 00}^{2}}\right], \\
\left(r>r_{0}\right) .
\end{array}
$$

Applying the Fresnel-Kirchhoff diffraction analysis, we have the following amplitude function $U_{1}$ at the first focal point:

$$
U_{1}(\rho, Z)=\frac{2 \pi \Gamma(\rho, Z)}{\lambda_{s} L^{\prime}} \int J_{0}\left(Z_{0}\right) U_{0}(r) \exp \left(\frac{i k_{s} r^{2} Z}{2 L^{\prime}}\right) \exp \left[-i k_{s} W(r)\right] r d r
$$

with

$$
\Gamma(\rho, Z)=\exp \left(-i k_{s} Z\right) \exp \left[-\frac{i k_{s}}{2 L^{\prime}}\left(X^{2}+Y^{2}+Z^{2}\right)\right]
$$

where $\rho^{2}=X^{2}+Y^{2}, J_{0}\left(Z_{0}\right)$ is the zeroth Bessel function with $Z_{0}=k_{s} r \rho / L^{\prime}, k_{s}=2 \pi / \lambda_{s}$, and $L^{\prime}$ is the distance between the lens and the focal point. The intensity distribution of the profile and the integrated intensity are given by

$$
I_{1}(\rho, Z)=\left|U_{1}(\rho, Z)\right|^{2}, \quad S_{1}(Z)=\int I_{1}(\rho, Z) \rho d \rho .
$$

The function $U_{2}(\rho, Z)$ at the second focal point is given by

$U_{2}(\rho, Z)=\frac{(2 \pi)^{2} \Gamma(\rho, Z)}{\left(\lambda_{s} L^{\prime}\right)^{2}} \iint U_{1}(r) \exp \left[\frac{i k_{s} \rho^{2}}{2 L^{\prime}}\left(1+\frac{Z}{L^{\prime}}\right)\right] J_{0}\left(Z_{1}\right) J_{0}\left(Z_{2}\right) \exp \left(\frac{i k_{s} r^{2} Z}{2 L^{\prime 2}}\right) \exp \left[-i k_{s} W(r)\right] r d r \rho d \rho$,

where $Z_{1}=k_{s} r \rho / L^{\prime}$ and $Z_{2}=\left(k_{s} r \rho / L^{\prime}\right)\left(1+Z / L^{\prime}\right)$.

Applying Fourier transformation on the function $U_{n}(X, Y, Z=0) \Gamma(X, Y, Z=0)^{*}$, we get

$$
F_{n}\left(k_{x}, k_{y}\right)=\left|P\left(u^{\prime}, v^{\prime}\right)\right| \exp \left[-i n k_{s} W\left(u^{\prime}, v^{\prime}\right)\right] U_{0}\left(u^{\prime}, v^{\prime}\right),
$$

where $u^{\prime}=k_{x} L^{\prime} / k_{s}, v^{\prime}=k_{y} L^{\prime} / k_{s}$, and $|P(u, v)|$ is the pupil function of the lenses, which is given by $|P(u, v)|=$ 1 for $r_{1}<\left(u^{2}+v^{2}\right)^{1 / 2}<r_{2}$ and 0 for others.

The function $U_{n}(X, Y, 0)$ at the $n$th focal point can be obtained by the inverse transformation. Equation (4) indicates that the aberration effects on the profiles are accumulated algebraically. In addition, we have generally the following relation:

$$
\iint\left|F_{n}\left(k_{x}, k_{y}\right)\right|^{2} d k_{x} d k_{y}=\iint\left|U_{n}(X, Y, 0)\right|^{2} d X d Y .
$$

Substituting $F_{n}\left(k_{x}, k_{y}\right)$ of Eq. (4) into the above equation, we find that the integrated intensity of the profile is conserved through the lens series even if there are aberrations.

The focal strength of the first lens is half of that of the following lenses downstream. Dividing each lens into two parts except for the first lens, we have a completely periodic structure with symmetric unit cells. Since the focal points are at the center of each cell, a symmetric amplitude function is expected in all the cells in the case of no aberration, which implies no beam loss in that case. In the presence of aberration, we also expect no beam loss by the consideration of Fourier transformation. 


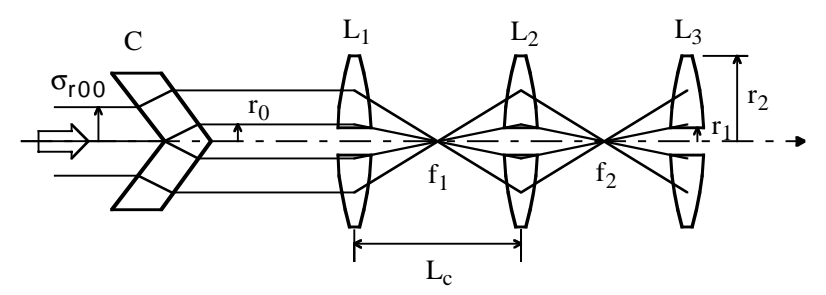

FIG. 3. Initial part of the optical system. A Gaussian laser beam is converted by a circular cone to a hollow beam and focused periodically in lens series.

\section{Numerical results}

Figure 4 shows the profiles, normalized by the peak value at $X=Z=0$, at the first focal point for various $Z$ in the case of no aberration of the first lens or $\xi_{1}=0$. The profiles are symmetric for $\pm X$ because of axial symmetry, represent nearly Gaussian distribution for all $Z$, and decrease nearly to zero around $X \approx 80 \mu \mathrm{m}$. As shown by various lines, profiles can be fitted well by

$$
I_{n}(X)=a(Z)+b(Z) \exp \left[-\frac{X^{2}}{2 \sigma_{r}(Z)^{2}}\right] .
$$

The parameters $a(Z), b(Z)$, and $\sigma_{r}(Z)$ against $Z$ are shown in Fig. 5. The parameters are symmetric for $\pm Z$, and beam size $\sigma_{r}(Z)$ has its minimum $\sigma_{r 0}=26 \mu \mathrm{m}$ at $Z=0$, and increases gradually with $Z$. The peak value $I_{n p}(Z)$ for different $Z$ is nearly equal to $b(Z)$ since $a(Z)$ is very small. The parameters are fitted further by the polynomials of $Z$ up to the ninth power, as shown with solid lines, which are used for the calculation of the collision rate.

Figure 6 shows the parameters at the focal point in the presence of aberration $\xi_{1}=9.06 \times 10^{-4}$, which is the magnitude for a conventional lens as given above. The figure indicates that the beam sizes are nearly the same as

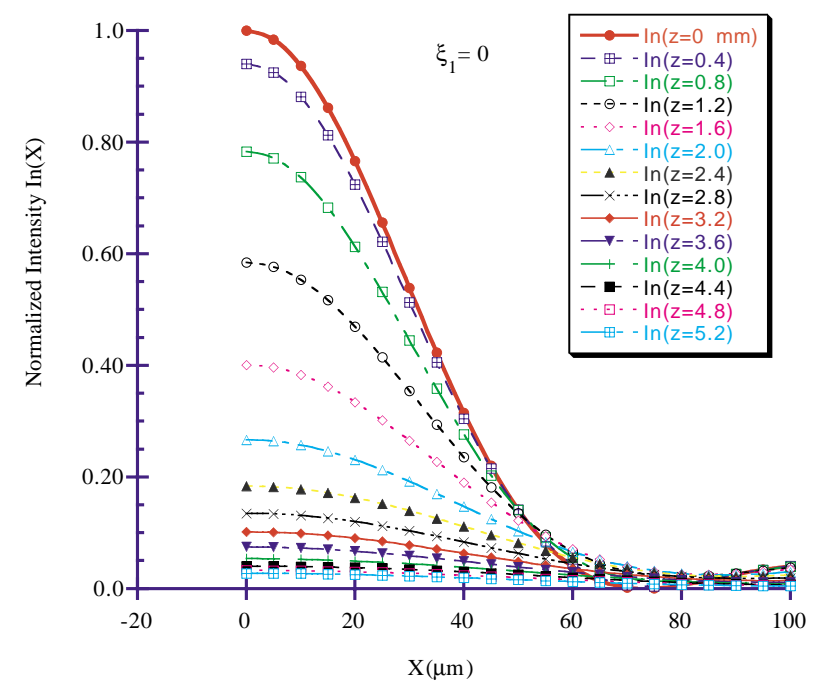

FIG. 4. (Color) Normalized laser beam profiles at the first focal point for no aberration.

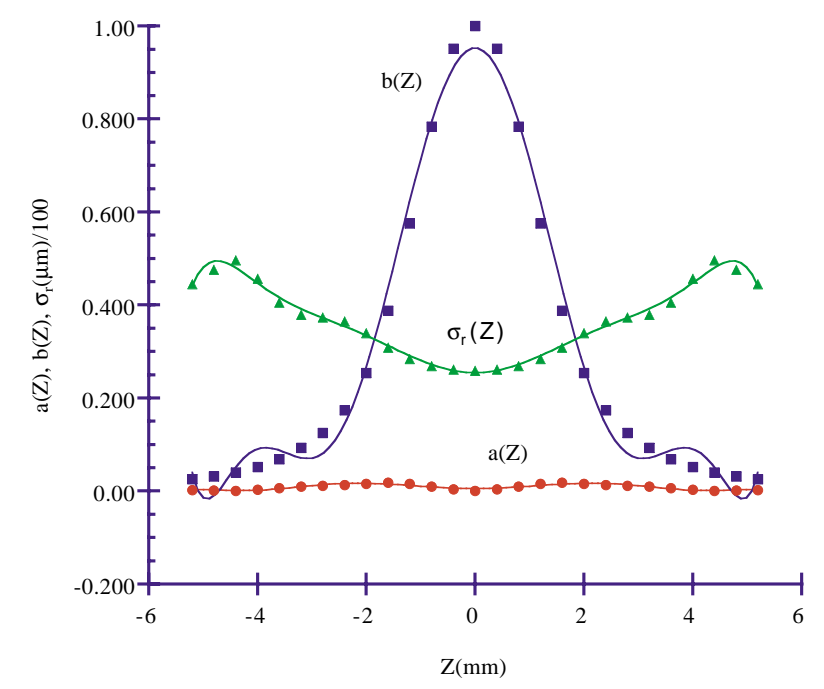

FIG. 5. (Color) Parameters $a(Z), b(Z)$, and $\sigma_{r}(Z)$ obtained by the fitting to the laser beam profiles for no aberration.

those for no aberration, but the minimum position of $\sigma_{r}(Z)$ is shifted to $Z \approx 2.8 \mathrm{~mm}$.

Characteristic features of the profiles at the second focal point can be observed by the peak values $I_{n p}(Z)$, as shown in Fig. 7. The peak values for no aberration and those for $\xi_{1}=9.06 \times 10^{-4}$ and $\xi_{2}=0$ are almost the same as those at the first focal point, where $\xi_{2}$ is the aberration coefficient of the second lens. In the case of $\xi_{1}=\xi_{2}=$ $-4.53 \times 10^{-4}$, the peak values are the same as those at the first focal point for $\xi_{1}=9.06 \times 10^{-4}$, but reversed with respect to $Z=0$. Thus the aberration effects are accumulated.

The integrated intensity for $\rho=0 \sim 5 \mathrm{~mm}$ is $S_{1}=0.99979$ and $S_{2}=0.99941$ at the first and the second focal point, respectively, in the case of no aberration. In the presence of aberration, $\xi_{1}=9.06 \times 10^{-4}$ and

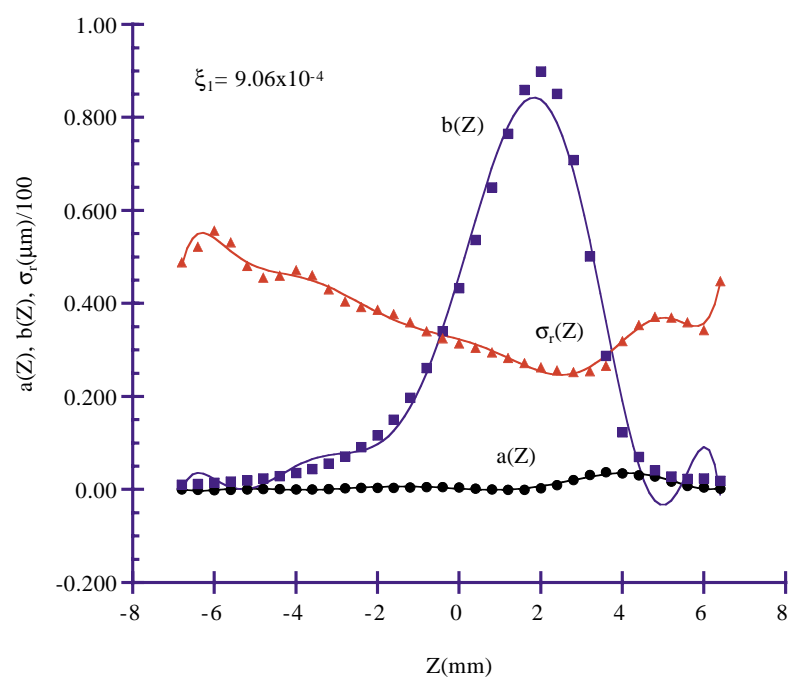

FIG. 6. (Color) Parameters $a(Z), b(Z)$, and $\sigma_{r}(Z)$ in the presence of aberration with $\xi_{1}=9.06 \times 10^{-4}$. 


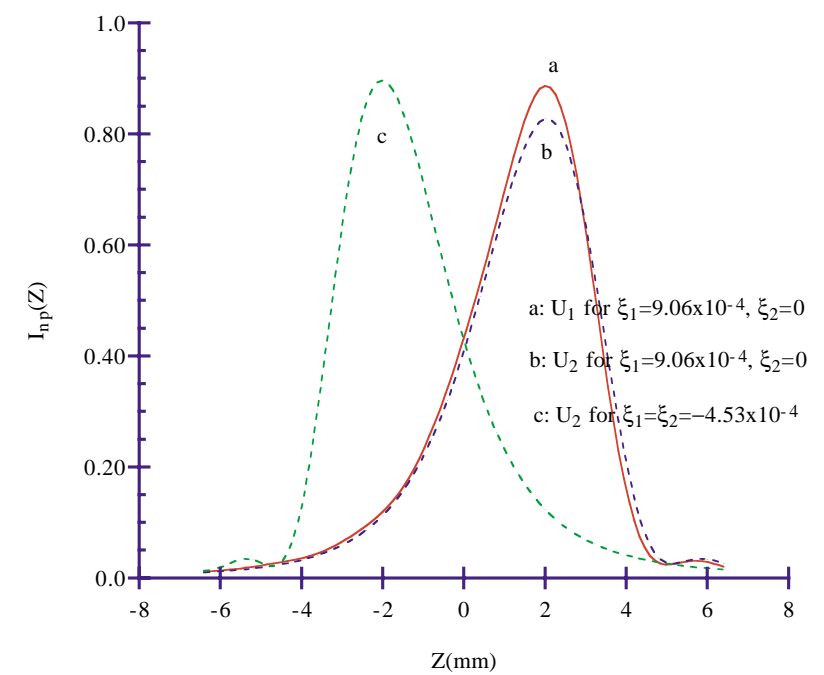

FIG. 7. (Color) Peak value $I_{n p}(Z)$ of normalized profile intensity. The solid line represents $I_{n p}(Z)$ of the profiles at the first focal point for the aberration $\xi_{1}=9.06 \times 10^{-4}$ and $\xi_{2}=0$. The broken lines represent $I_{n p}(Z)$ at the second focal point for $\xi_{1}=$ $9.06 \times 10^{-4}$ with $\xi_{2}=0$ and $\xi_{1}=\xi_{2}=-4.53 \times 10^{-4}$.

$-9.06 \times 10^{-4}$, we have $S_{2}=0.99945$ and 0.99938 , respectively, which are almost the same as that for no aberration. Therefore, the beam loss by diffraction is negligibly small even in the presence of aberration.

\section{ELECTRON BEAM FOCUSING}

The electron beam with an energy of $5.8 \mathrm{GeV}$ is focused by a superconducting solenoid with a field $B_{s}=15 \mathrm{~T}$. The beam is first focused by quadrupole magnets in the injection beam line to a minimum size $\sigma_{x 0}=\sigma_{y 0}$ at the entrance of the solenoid. The electron beam size in the field for the beam emittance $\varepsilon_{x}=8.81 \times 10^{-10} \mathrm{rad} \mathrm{m}$ is expressed as

$$
\sigma_{x}(z)=\left\{\sigma_{x 0}^{2}+\left[\left(\alpha \sigma_{x 0}^{\prime}\right)^{2}-\sigma_{x 0}^{2}\right] \sin ^{2} \phi\right\}^{1 / 2},
$$

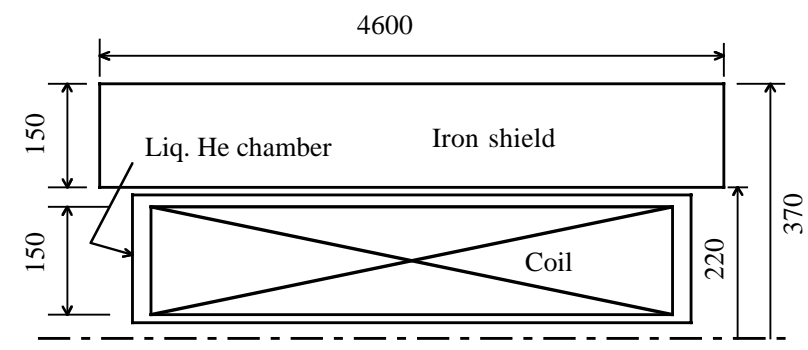

FIG. 8. Rough dimensions of a superconducting solenoid.

with $\sigma_{x 0}^{\prime}=\varepsilon_{x} / \sigma_{x 0}$ and $\alpha=v_{z} / \omega=m_{0} \gamma v_{z} / e B_{s}$, where $m_{0}$ and $e$ are the rest mass and charge of electron, $\gamma$ is the relativistic factor of beam energy, $v_{z}$ is the longitudinal velocity of the beam, $\omega$ is the cyclotron frequency, and $\phi=\omega z / v_{z}$. Then the beam size is kept constant in the field at $\alpha=\sigma_{x 0} / \sigma_{x 0}^{\prime}$ or at $\sigma_{x 0}=34 \mu \mathrm{m}$. Rough dimensions of the solenoid are shown in Fig. 8, where an iron shield is used, if necessary, to suppress a stray field. Since the critical current of the superconducting wire made of $\mathrm{Nb}_{3} \mathrm{Sn}$ is not very high in such a high field, a large amount of wire is needed for the solenoid.

\section{IV. $\gamma$-RAY YIELD}

The electron beam and the laser pulse are assumed to have a Gaussian distribution with a length of $\sigma_{z}$ and $\sigma_{s z}$, respectively. If the transverse sizes are constant with $\sigma_{x 0}\left(=\sigma_{y 0}\right)$ and $\sigma_{r 0}$, and the collision rate is given by

$$
N_{t 0}=\frac{n_{e} n_{s} \sigma_{T}}{2 \pi\left(\sigma_{x 0}^{2}+\sigma_{r 0}^{2}\right)},
$$

where $n_{e}$ and $n_{s}$ are the number of electrons and photons per bunch and pulse, respectively, and $\sigma_{T}$ is the cross section for Thomson scattering.

Taking into account the variation of the laser beam size along the $Z$ axis, we have the following collision rate $N_{t}=$ $N_{a}+N_{b}$ between one bunch and one pulse:

$$
\begin{aligned}
& N_{a}=\frac{n_{e} n_{s} \sigma_{T}}{(2 \pi)^{2} \sigma_{z} \sigma_{s z}} \iint \frac{a(Z)}{\sigma_{r}(Z)^{2}} \exp \left(-\frac{z^{2}}{2 \sigma_{z}^{2}}-\frac{z^{\prime 2}}{2 \sigma_{s z}^{2}}\right) d z d z^{\prime} \\
& N_{b}=\frac{n_{e} n_{s} \sigma_{T}}{(2 \pi)^{2} \sigma_{z} \sigma_{s z}} \iint \frac{b(Z)}{\sigma_{z}^{2}+\sigma_{r}(Z)^{2}} \exp \left(-\frac{z^{2}}{2 \sigma_{z}^{2}}-\frac{z^{\prime 2}}{2 \sigma_{s z}^{2}}\right) d z d z^{\prime}
\end{aligned}
$$

where $Z=\left(z+z^{\prime}\right) / 2$, and $z$ and $z^{\prime}$ are taken from the center of the electron bunch and laser pulse, respectively.

A reduction factor $F_{r}$ of collision rate is introduced by $N_{t}=F_{r} N_{t 0}$. Numerically, we have $F_{r}=0.60$ for $\sigma_{z}=$ $0.9 \mathrm{~mm}$ and $\sigma_{s z}=3 \mathrm{~mm}$ for no aberration, and $F_{r}=$ 0.41 for $\xi_{1}=9.06 \times 10^{-4}$. Thus, the collision rate is considerably affected by such an aberration.

A lens, made of $\mathrm{ZnSe}$, has a refraction index of 2.4028, a bulk absorption coefficient smaller than $0.05 \% / \mathrm{cm}$, and a reflectivity smaller than $0.20 \%$ by special coating.
For a matched collision between the electron bunches and the laser pulses at the focal points, the distances of bunches and pulses are both $2 L_{c}$. To make the collision $N_{c}$ times with each electron bunch, we need a laser pulse number $N_{p}=N_{b}+N_{c}-1$ per train, where $N_{b}$ is the number of electron bunches per train. The $\gamma$-ray yield is given by

$$
Y=\frac{n_{e} n_{s 0} \eta N_{b} N_{c} f_{r} \sigma_{T} F_{r}}{2 \pi\left(\sigma_{x 0}^{2}+\sigma_{r 0}^{2}\right)}
$$


TABLE I. Parameters of Compton backscattering for polarized $\gamma$-ray generation.

\begin{tabular}{lcc}
\hline \hline \multicolumn{1}{c}{ Electron beam } & Proposal I & This work \\
\hline Beam energy $E(\mathrm{GeV})$ & & \\
Normalized emittance $\varepsilon_{x 0}(\mathrm{~mm}$ mrad $)$ & 10 & 5.8 \\
Number of electrons $n_{e}(/$ bunch $)$ & $10^{11}$ & 10 \\
Number of bunches $N_{b}(/$ train $)$ & 85 & 85 \\
Bunch length $\sigma_{z}(\mathrm{~mm})$ & 0.9 & 0.9 \\
Beam size $\sigma_{x 0}(\mu \mathrm{m})$ & 21 & 34 \\
\multicolumn{1}{c}{$\mathrm{CO}_{2}$ laser beam } & & \\
Wavelength $\lambda_{s}(\mu \mathrm{m})$ & 10.6 & 10.6 \\
Pulse length $\sigma_{s z}(\mathrm{~mm})$ & 3 & $3(0.9)$ \\
Beam size $\sigma_{r 0}(\mu \mathrm{m})$ & 15.5 & 26 \\
Pulse energy $E_{J}(\mathrm{~J})$ & 0.25 & $1.5(1.0)$ \\
Number of pulses $N_{p}(/$ train $)$ & 85 & 104 \\
Laser power $P_{L}(\mathrm{~kW})$ & 3.2 & $24(16)$ \\
Number of lasers $N_{L}$ & 40 & 1 \\
$\quad$ Compton backscattering & & \\
Maximum $\gamma$-ray energy $\varepsilon_{\gamma}(\mathrm{MeV})$ & 60 & 60 \\
Repetition frequency $f_{r}(\mathrm{~Hz})$ & 150 & 150 \\
$\gamma$-ray yield $Y(\gamma / \mathrm{s})$ & $7 \times 10^{15}$ & $7 \times 10^{15}$ \\
Number of cells $N_{c}$ & 40 & 20 \\
Reduction factor $F_{r}$ & $\ldots$ & $0.60(0.87)$ \\
\hline \hline
\end{tabular}

where $n_{s 0}$ is the initial photon number per pulse, $\eta$ $(=0.97)$ is the average transmission factor due to the lens loss by the absorption and reflection, and $f_{r}$ is the repetition frequency. In order to get the required $\gamma$-ray yield, we need $n_{s 0}=7.9 \times 10^{19}$ photons per pulse or a pulse energy of $E_{J}=1.5 \mathrm{~J}$. Accordingly, a laser power $P_{L}=24 \mathrm{~kW}$ with $N_{p}=104$ pulses per train is needed. The heat load in a lens is about $6 \mathrm{~W}$. If the laser pulse length is reduced to $\sigma_{s z}=0.9 \mathrm{~mm}$, we have $F_{r}=0.87$, so that the required laser power is decreased to $P_{L}=16 \mathrm{~kW}$. The parameters of the present work are summarized in Table I and compared with Proposal I.

\section{DISCUSSION AND CONCLUSION}

We considered the case $r_{0}=r_{1}=3 \mathrm{~mm}$ for a critical discussion. If we set $r_{0}>r_{1}$, we have a safety margin to avoid a beam loss which might be induced by accidental and construction errors. Some of the scattered photons with an energy lower than $1 \mathrm{MeV}$ collide the lenses by passing through the holes, the radiation power of which amounts to about $9 \mathrm{~W}$ in the end lens. Investigation of radiation effects on the lenses is required. If serious damage is induced, it is necessary to increase the hole size and install radiation shields.

In this paper, discussions on the basic concept of lens series for an intense $\gamma$-ray generation were presented. The laser beam loss in the lens series is considered to be negligibly small, and we can significantly reduce the laser power required for the $\gamma$-ray yield compared with Proposal I. Furthermore, it is expected that the laser power can be reduced to several $\mathrm{kW}$ if the number of unit cells in the lens series is increased to about 100 along with the increase of the aperture and hole size of the lenses, which is discussed elsewhere. It is valuable to make a further investigation on the present system for technical practice. This method can be applied to a similar system for enhancing the collision rate.

[1] V.E. Balakin and A. A. Mikhailichenko, Budkar Institute of Nuclear Physics Report No. INP 79-85, 1979.

[2] E. G. Bessonov, in Proceedings of the 15th International Conference on High Energy Accelerators, Hamburg, 1992 (World Scientific, London, 1993), Vol. 1, p. 138.

[3] T. Omori, K. Dobashi, T. Hirose, T. Kimura, Y. Kurihara, T. Okugi, K. Sugiyama, A. Tsunemi, and M. Washio, TMU-HEP-EXP-98-10. 